\title{
A Study on VRSA Prevalence in Hospital Settings- Bangalore India
}

\section{Mandhri Abeysooriya* and Cotek Temitayo}

Department of Microbiology and Biochemistry, Indian Academy Degree College Autonomous, Centre for Research and Post Graduate Studies, Bangalore, Karnataka, India

*Corresponding Author: Mandri Abeysooriya, Department of Microbiology and Biochemistry, Indian Academy Degree College Autonomous, Centre for Research and Post Graduate Studies, Bangalore, Karnataka, India.

Received: July 10, 2019; Published: August 09, 2019

DOI: $10.31080 /$ ASMI.2019.02.0330

\begin{abstract}
Objective: Staphylococcus aureus (SA) is one of the most common pathogenic Gram-positive bacteria. In this study, SA isolated from three different hospitals in Bangalore, India were tested against various antibiotics including vancomycin, cefoxitin, co-trimoxazole and ciprofloxacin for their sensitivity, especially against vancomycin to show the presence of vancomycin resistance $S$. aureus (VRSA) in clinical samples.

Methods: A total number of 25 samples from gauze bandage of skin lesions and wounds dressings were collected from various hospitals in Bangalore. The samples included pus swabs from skin lesions, swabs or open wounds from treated patients. These samples were inoculated into selective media for S. aureus (MSA-Mannitol salt agar) and 37 morphologically SA isolates were recovered. These putative SA isolates were then further characterized using traditional biochemical tests including Catalase, Oxidase, Coagulase and Mannitol fermentation tests. Subsequently, each Staphylococcus aureus isolates was tested against antibiotics for their susceptibility. Results: A total of 37 isolates were characterized using the traditional biochemical test methods and 16 were identified as $S$. aureus. Antimicrobial resistance profiling using minimum inhibitory concentration (MIC) measurements showed that eight isolates of SA were resistant to vancomycin. and five isolates were resistant to ciprofloxacin.

Conclusion: Vancomycin resistant SA (VRSA) is becoming more prominent in hospitals in Bangalore, India.
\end{abstract}

Keywords: Staphylococcus aureus; VRSA; Vancomycin; Ciprofoxacin

\section{Introduction}

Staphylococcus aureus (SA) is among the most common pathogens isolated from skin, eyes, cerebrospinal fluid, blood, respiratory and gastrointestinal tract, bone, and connective tissues. $S$. aureus is the leading cause of community acquired skin and soft tissue infections such as abscesses (boils), furuncles and cellulitis. Skin infections are common, but bacteria can spread through the bloodstream infect distant organs [1]. Surgical site infections are a serious nosocomial infection and significantly contribute to patient morbidity and healthcare costs. When disseminated, SA is responsible for diverse conditions such as toxic shock syndrome, osteomyelitis, meningitis, bacterial endocarditis. SA septicemia may enhance the mortality rate up to $40 \%$ [2]. Spread is by direct contact with an infected person, by a contaminated object, or by inhaling infected droplets dispersed by sneezing or coughing.

Many strains have developed resistance to antibiotics. Genetically bacteria use two main strategies to adopt for the antibiotic activity; i. De novo mutation in the genome where it interferes with the mechanism of activity of the compound, ii. Exchange of the for- 
eign DNA containing resistant determinants via horizontal gene transfer (HGT) [3]. De novo mutations can alter target sites causing them to be insensitive to the action of antibiotics or causes activation of latent resistant mechanisms. One frequent de novo mutation to be observed is mutations in penicillin binding proteins which renders them to resistant to beta lactams. Moreover, some antibiotic efflux pumps are repressed at the transcription level. Mutations in repressor proteins activate efflux pump expression resulting in broad spectrum antimicrobial resistance. SA uses all three methods of HGT: Conjugation, Transformation and Transduction.

Beta lactams are drug of choice for treating bacterial infections due to their low toxicity and high therapeutic indices. SA uses few mechanisms to combat the action of beta lactams. Beta lactamases are enzymes that open the lactam ring in the active site of the drug and render them inactive. Plasmid bourn or chromosomal located beta lactamase BlaZ confer resistance to broad spectrum of betalactamses such as first and second generation cephalosporins and commonly used penicillins such as benzyl penicllins and ampicillin derivatives and second or third generation penicillins. The advent of Beta lactamase resistant penicillins such as methicillin and its derivatives against SA was a key event in therapy but due to their heavy use, methicillin resistant SA (MRSA) became a problem in both nosocomial and community acquired SA infections. Methicillin resistance is due to the presence of a heterologous horizontally acquired homolog of penicillin binding protein 2 (PBP2) which cannot be inhibited by beta lactams.

Centers for Disease Control and Prevention (CDC) reported a prevalence of SA in hospital infections of about 18\% [4]. Asia has been reported as a continent which has higher number of MRSA in the world [5]. Vancomycin-intermediate S. aureus (VISA) strains and vancomycin-resistant $S$. aureus (VRSA) strains are also being increasingly identified in certain countries in this region [6,7]. A fast-growing number of VRSA mostly evolved from MRSA has also emerged and thus both Methicillin and vancomycin [8,9]. It was identified that excess accumulation of peptidoglycan which thicken the cell wall due to unidentified mutations paves the way for the resistance against vancomycin by $S$. aureus making it's the most common resistance mechanism for VRSA strains [10].

MRSA infection that is acquired in a hospital is treated with antibiotics that are effective against MRSA. MRSA infections can be treated with less toxic antibiotics such as trimethoprim-sulfamethoxazole, 4-amino quinolones or cephalosporins which are relatively cheaper and can be orally administered. Vancomycin and daptomycin are the first-line therapies for MRSA bacteremia if drug resistance profiles indicates other simpler and cheaper antimicrobials are useless against MRSA [11]. MRSA isolates that are also multidrug resistant and also resistant to vancomycin are treated with antimicrobials which are recently developed or expensive. Some of these are linezolids, tedizolids, quinupristin plus dalfopristin, ceftaroline, telavancin, or daptomycin. Daptomycin, which is a lipopeptide with a poorly characterized antimicrobial mechanism has become the main stay therapy against MRSA which are also resistant to vancomycin.

Two shifts in epidemiology of SA can be witnessed in last past two decades: first, a growing number of health care-associated infections, particularly seen in infective endocarditis and prosthetic device infections, and second, an epidemic of community-associated skin and soft tissue infections driven by strains with certain virulence factors and resistance to $\beta$-lactam antibiotics [12]. On the other hand, Ciprofloxacin has been used invitro experiments in treating against VRSA [13] but further research is ongoing on whether it could be a better option on treating VISA and VRSA strains. Considering the recent studies showing the emergence of VRSA, we determined the prevalence of VISA and VRSA strains in three different hospitals in Bangalore, India and additionally compared the resistance profile between vancomycin and Ciprofloxacin.

\section{Materials and Methods}

\section{Sample collection and isolation of Staphylococcus aureus}

A total number of 25 samples from different gauze bandages with skin lesions and wounds were collected from various hospitals in Bangalore which includes Cratis hospital, Avietha and Chris hospital. Swabs of pus from wounds and surgical sites were collected after dressings. Aseptic plating on nutrient agar was done for all samples in the laboratory. About 37 different isolates was derived from the initial plates using colony morphology and Gram staining morphological characteristics.

\section{Mannitol fermentation test and other biochemical tests}

All 37 Gram-positive isolates were plated in Mannitol Salt agar (a selective and differential media for SA) and incubated in 37 degrees Celsius for 24 hours. The observation of growth and color change from red to yellow indicates fermentation of Mannitol by the bacterial culture. The $\mathrm{pH}$ indicator used is phenol red, therefore the positive color change from red to yellow indicates acid produc- 
tion and $\mathrm{pH}$ change in the media by bacteria. Subsequently, further biochemical tests were carried out which included catalase, oxidase, Indole, Methyl red, Voges-Proskauer, Citrate utilization test (IMViC) and Coagulase test.

\begin{tabular}{|l|c|c|c|}
\hline Test & Medium & Reagent & Result \\
\hline Indole & Tryptophan broth & $\begin{array}{c}\text { Ko Vac's re- } \\
\text { agent }\end{array}$ & Positive \\
\hline $\begin{array}{l}\text { Voges-Pros- } \\
\text { kauer }\end{array}$ & $\begin{array}{c}\text { Voges-Proskauer } \\
\text { broth }\end{array}$ & $\begin{array}{c}\text { Barritt's A and } \\
\text { reagent } \\
\text { Barritt's B }\end{array}$ & Positived \\
\hline Citrate & $\begin{array}{c}\text { Simmons citrate } \\
\text { agar }\end{array}$ & Positive \\
\hline Catalase & $\begin{array}{c}\text { Saline solution } \\
\text { Oxidase disc }\end{array}$ & $\begin{array}{c}\text { Hydrogen Per- } \\
\text { oxide }\end{array}$ & Positive \\
\hline $\begin{array}{l}\text { Oxidase } \\
\text { Fermenta- } \\
\text { tion }\end{array}$ & Mannitol Salt agar & phenol red & Positive \\
\hline Coagulase & Blood Agar media & Negative \\
\hline
\end{tabular}

Table 1: Summary of biochemical tests that were carried out to differentiate SA from other Gram-positive cocci.
Bacterial antimicrobial sensitivity tests.

All samples were plated on Muller Hinton agar media and grown for antimicrobial resistance profiling. To determine vancomycin resistance, disc diffusion method using vancomycin discs (Va30 Hi-media) was used. Moreover, using disc diffusion method, four other laboratory antibiotics including cefoxitin (Cx30), ketoconazole (Kt30), co-Trimoxazole (Cot25) and ciprofloxacin (Cip5) were tested against SA isolates for the MIC determination. Measurement of each zone of growth inhibition was measured and the diameter was recorded as millimeters for MIC determination.

\section{Results and Discussion}

In this study, a total of 25 samples were taken from three different hospitals in Bangalore, India. 37 isolates were derived from these samples and sixteen (48.6\%) were identified as SA using variety of biochemical tests (Table 2). MIC determination indicated that eight (50\%) were resistant to vancomycin while the other half (51.4\%) were susceptible to vancomycin (Table 3). Further testing with 4 other antimicrobials showed $100 \%$ resistance to cefoxitin (Cx30), ketoconazole (Kt30) and Co-Trimoxazole (Cot25), but surprisingly, five of the SA isolates that are VRSA showed sensitive to Ciprofloxacin (Cip5).

\begin{tabular}{|c|c|c|c|c|c|c|c|c|c|}
\hline $\begin{array}{l}\text { Bacterial } \\
\text { isolates }\end{array}$ & Media & $\begin{array}{c}\text { Citrate } \\
\text { test }\end{array}$ & $\begin{array}{c}\text { Indole } \\
\text { test }\end{array}$ & $\begin{array}{l}\text { Vogues Pr } \\
\text { test }\end{array}$ & $\begin{array}{l}\text { Methyl } \\
\text { red test }\end{array}$ & $\begin{array}{l}\text { Oxidase } \\
\text { test }\end{array}$ & $\begin{array}{c}\text { Catalase } \\
\text { test }\end{array}$ & Coagulase & $\begin{array}{c}\text { Mannitol } \\
\text { fermentation }\end{array}$ \\
\hline $\mathrm{CH} 1$ & N.A, MSA & - & - & + & - & + & - & - & - \\
\hline CH 10-3 & N.A, MSA & - & + & - & + & + & + & - & - \\
\hline CH 10-4 & N.A, MSA & - & + & - & - & + & - & - & - \\
\hline $\mathrm{CH} 3$ & N.A, MSA, Blood Agar & + & - & + & + & - & + & + & + \\
\hline A & N.A, MSA, Blood Agar & + & - & + & + & - & + & + & + \\
\hline AV1 10-3N & N.A, MSA & + & - & + & + & + & + & - & - \\
\hline AV1 10-3M & N.A, MSA, Blood Agar & + & - & + & + & - & + & + & + \\
\hline AV1 10-4M & N.A, MSA, Blood Agar & + & - & + & + & - & + & + & + \\
\hline AV2 10-3 & N.A, MSA & - & + & - & - & + & - & - & - \\
\hline AV3 10-3 & N.A, MSA & - & - & - & + & + & - & - & - \\
\hline AV2 10-4 & N.A, MSA & - & + & - & - & + & - & - & - \\
\hline CR1 10-3 M & N.A, MSA, Blood Agar & + & - & + & + & - & + & + & + \\
\hline CR1 10-4 M & N.A, MSA & - & + & + & + & + & + & - & + \\
\hline CR2 10-3 & N.A, MSA & - & - & - & - & + & - & - & + \\
\hline CR2 10-4 & N.A, MSA, Blood Agar & + & - & + & + & - & + & + & + \\
\hline
\end{tabular}




\begin{tabular}{|c|c|c|c|c|c|c|c|c|c|}
\hline CR3 10-3 & N.A, MSA & + & - & - & - & + & - & - & - \\
\hline CR4 & N.A, MSA & + & + & + & - & - & + & - & - \\
\hline CR5 & N.A, MSA & + & + & + & - & + & - & - & - \\
\hline CR6 & N.A, MSA, Blood Agar & + & - & + & + & - & + & + & + \\
\hline CR7 & N.A, MSA & + & + & + & - & + & - & - & - \\
\hline CR8 & N.A, MSA & - & + & + & - & + & - & - & - \\
\hline B1CR4 & N.A, MSA & - & + & - & - & + & - & - & - \\
\hline B2CR5 & N.A, MSA & - & + & - & - & + & + & - & - \\
\hline B3CR6 & N.A, MSA & + & - & + & + & + & - & - & - \\
\hline B4CR7 & N.A, MSA, Blood Agar & + & - & - & - & - & + & + & + \\
\hline B5CR8 & N.A, MSA, Blood Agar & + & - & + & + & - & + & + & + \\
\hline B6CR9 & N.A, MSA, Blood Agar & + & - & + & + & - & + & + & + \\
\hline B7CR1 & N.A, MSA & - & + & - & - & + & + & + & - \\
\hline CR11 & N.A, MSA, Blood Agar & + & - & + & + & - & + & + & + \\
\hline CR12 & N.A, MSA & - & + & - & - & + & - & + & - \\
\hline CR13 & N.A, MSA, Blood Agar & + & - & - & + & - & + & + & + \\
\hline CR14 & N.A, MSA, Blood Agar & + & - & + & + & - & + & + & + \\
\hline CR15 & N.A, MSA, Blood Agar & + & - & + & + & - & + & + & + \\
\hline CR16 & N.A, MSA, Blood Agar & + & - & + & + & - & + & + & + \\
\hline UK & N.A, MSA, Blood Agar & + & - & + & + & - & + & + & + \\
\hline $10-3 \mathrm{M}$ & N.A, MSA & + & + & + & + & + & - & - & - \\
\hline $10-4 \mathrm{M}$ & N.A, MSA & - & + & - & - & + & - & - & - \\
\hline
\end{tabular}

Table 2: Biochemical test results for identification of SA strains in patient samples.

\begin{tabular}{|l|c|c|c|c|c|}
\hline $\begin{array}{l}\text { S. aureus } \\
\text { culture }\end{array}$ & $\begin{array}{c}\text { Vancomycin } \\
\text { (zone of inhibition) } \\
\text { in cm }\end{array}$ & $\begin{array}{c}\text { Cefoxitin Cx30 } \\
\text { zone of inhibition) } \\
\text { in cm }\end{array}$ & $\begin{array}{c}\text { Ketoconazole Kt30 } \\
\text { (zone of inhibition) } \\
\text { in cm }\end{array}$ & $\begin{array}{c}\text { Co-Trimoxazole Cot25 } \\
\text { (zone of inhibition) } \\
\text { in cm }\end{array}$ & $\begin{array}{c}\text { Ciprofloxacin Cip5 } \\
\text { (zone of inhibition) } \\
\text { in cm }\end{array}$ \\
\hline B5 & 0 & 0 & 0 & 0 & 1.3 \\
\hline b6 & 2 & 0 & 0 & 0 & 2.5 \\
\hline A & 0 & 0 & 0 & 0 & 0 \\
\hline Ch3 & 0 & 0 & 0 & 0 & 0 \\
\hline AV10-3m & 0 & 0 & 0 & 0 & 1.1 \\
\hline Cr10-3M & 1.6 & 0 & 0 & 0 & 1.6 \\
\hline Cr2.10-4 & 0 & 0 & 0 & 0 & 0 \\
\hline cr15 & 0 & 0 & 0 & 0 & 0 \\
\hline UK & 2.1 & 0 & 0 & 0 & 2 \\
\hline Cr16 & 1 & 0 & 0 & 0 & 1.6 \\
\hline Cr13 & 0 & 0 & 0 & 0 & 2 \\
\hline cr11 & 1.8 & 0 & 0 & 0 & 2.3 \\
\hline Av10-4m & 1.3 & 0 & 0 & 0 & 1.7 \\
\hline Cr6 & 1.1 & 0 & 0 & 0 & 2 \\
\hline Cr14 & 1.1 & 0 & 0 & 0 & 0 \\
\hline B4 & 0 & 0 & 0 & 0 & \\
\hline
\end{tabular}

Table 3: MIC results derived from disc diffusion assays. The bacterial clearing diameter is recorded in centimeters measured from the edge of the antimicrobial disc. 

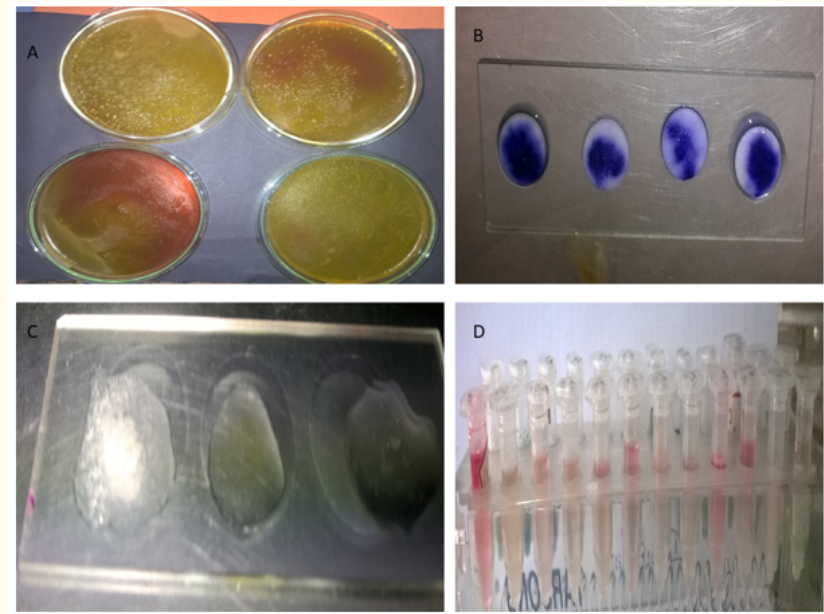

Figure 1: (A) Showing Mannitol fermentation test. (B) Shows oxidase test. (C) Shows Catalase test. (D) Shows oxidase test.

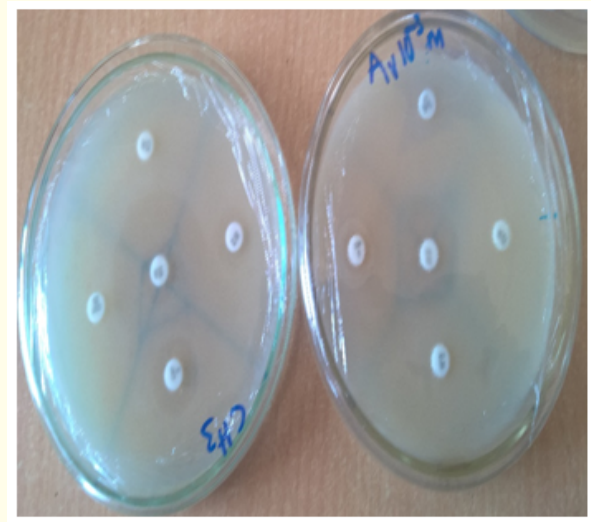

Figure 2: Plates showing clear zones of inhibition and no zones of inhibition.

\section{Conclusion}

This study shows prevalence of vancomycin resistance strain of Staphylococcus aureus from clinical isolates derived from hospitals in Bangalore, India. The high percentage (50\%) of VRSA strain shows that vancomycin use should be always proceeded by MIC testing for its efficacy. There wasn't a definite correlation between the hospital locality or that of the patients from the sampling en- viron. In comparison to previous years, the resistance of $S$. aureus strain to vancomycin has been increasing, indicating that thorough MIC determination against antimicrobials is paramount important in hospital practices. Moreover, we demonstrated that Ciprofloxacin could be an alternative antimicrobial against VRSA. Ciprofloxacin is relatively cheaper and orally administered and has less serious side effects than both vancomycin and daptomycin. This study should be followed up by a more extensive cross-sectional studies characterizing the antimicrobial resistance profiling using vancomycin, methicillin, daptomycin and ciprofloxacin to determine the MIC profiling of MRSA and VRSA strains. This study which demonstrated (i) vancomycin resistance has high prevalence and, (ii) some of vancomycin resistant strains are sensitive to quinolones could be the basis for a larger prevalence study in nation-wide study for recording VISA and VRSA strains in India.

\section{Acknowledgement}

The authors would like to acknowledge Indian Academy Degree College Autonomous, Bangalore, India, for the financial support and facilities for the success of this research work. We acknowledge Dr. Hepseeba K.V of Cratis Hospital Bangalore and the administrative of Chris and Avietha hospital, Bangalore India.

\section{Bibliography}

1. Adverse Health Events Reports and Fact Sheets - Minnesota Dept. of Health.

2. Sarrafzadeh F., et al. "Vancomycin-Resistant Staphylococcus aureus isolates among hospitalized patients; a tertiary medical care center experience from Southern Iran". Cogent Medicine 3 (2016): 1163768.

3. Munita JM and Arias CA. "Mechanisms of Antibiotic Resistance". Microbiology Spectrum 4 (2016).

4. Sievert DM., et al. "Antimicrobial-resistant pathogens associated with healthcare-associated infections: summary of data reported to the National Healthcare Safety Network at the Centers for Disease Control and Prevention, 2009-2010". Infection Control and Hospital Epidemiology 34 (2013): 1-14.

5. Chuang YY and Huang YC. "Molecular epidemiology of community-associated meticillin-resistant Staphylococcus aureus in Asia". Lancet Infection Disease 13 (2013): 698-708. 
6. Banerjee T and Anupurba S. "Colonization with VancomycinIntermediate Staphylococcus aureus Strains Containing the vanA Resistance Gene in a Tertiary-Care Center in North India”. Journal of Clinical Microbiology 50 (2012): 1730-1732.

7. Saha B., et al. "Identification and characterization of a vancomycin-resistant Staphylococcus aureus isolated from Kolkata (South Asia)". Journal of medical Microbiology 57 (2008): 7279.

8. Hasan R., et al. "Prevalence of vancomycin resistant Staphylococcus aureus (VRSA) in methicillin resistant S. aureus (MRSA) strains isolated from burn wound infections". Ci ji yi xue za zhi = Tzu-chi Medical Journal 28 (2016): 49-53.

9. Friãe A., et al. "Epidemiological survey of the first case of vancomycin-resistant Staphylococcus aureus infection in Europe". Epidemiology Infection 143 (2015): 745-748.

10. Mandell GL., et al. "Douglas, and Bennett's Principles and Practice of Infectious Diseases". (Churchill Livingstone Elsevier (2010).

11. Vardakas KZ., et al. "Carbapenems versus alternative antibiotics for the treatment of bacteraemia due to Enterobacteriaceae producing extended-spectrum $\beta$-lactamases: a systematic review and meta-analysis". Journal of Antimicrobial Chemotherapy 67 (2012): 2793-2803.

12. Davis JS., et al. "Combination of Vancomycin and $\beta$-Lactam Therapy for Methicillin-Resistant Staphylococcus aureus Bacteremia: A Pilot Multicenter Randomized Controlled Trial". Clinical Infectious Diseases 62 (2016): 173-180.

13. Smith SM and Eng RH. "Activity of ciprofloxacin against methicillin-resistant Staphylococcus aureus". Antimicrobial Agents and Chemotherapy 27 (1985): 688-691.

Volume 2 Issue 9 September 2019

(C) All rights arereserved by Mandhri Abeysooriya and Cotek Temitayo. 